\title{
A Hankel Transform Approach to Inverse Quasi-Static Steady-State Thermal Stresses in a Thick Circular Plate
}

\author{
Manoj P. Tripathi ${ }^{2}$ • Om P. Singh ${ }^{1}$
}

Published online: 7 August 2015

(C) Springer India Pvt. Ltd. 2015

\begin{abstract}
In this paper, we solve the realistic problem of inverse quasi-static steady-state thermal stresses in a thick circular plate, which is subjected to arbitrary interior temperature and determine the unknown temperature and thermal stresses on the upper surface of the thick circular plate, where the fixed circular edge and the lower surface of the circular plate are thermally insulated using Hankel transform. To achieve our objective, first we construct a new stable algorithm for numerical evaluation of Hankel transform of order $v>-1$. The integrand $r f(r) J_{v}(p r)$ consists of a slowly varying component $r f(r)$ and a rapidly oscillating component $J_{v}(p r)$. Most of the algorithms proposed in last few decades approximate the slowly varying component $r f(r)$. In the present paper, we take a different approach and replace the rapidly oscillating component $J_{v}(p r)$ in the integrand by its hat functions approximation. This approach avoids the complexity of evaluating integrals involving Bessel functions. This leads to a very simple, efficient and stable algorithm for numerical evaluation of Hankel transforms. We further give error and stability analysis and corroborate our theoretical findings by various numerical experiments.
\end{abstract}

Keywords Thermal stresses - Thick circular plate $\cdot$ Hankel transform · Hat basis functions Error analysis $\cdot$ Random noise

Mathematics Subject Classification $41 \mathrm{~A} 10 \cdot 44 \mathrm{~A} 15 \cdot 65 \mathrm{R} 10$

\footnotetext{
Om P. Singh

opsingh.apm@iitbhu.ac.in; singhom@gmail.com

Manoj P. Tripathi

mptripathi74@gmail.com

1 Department of Mathematical Sciences, Institute of Technology, Banaras Hindu University, Varanasi 221005, India

2 Department of Mathematics, Udai Pratap Autonomous College, Varanasi 221002, India
} 


\section{Introduction}

For the sake of continuity, we give a brief background of the difficulties in numerical evaluation of Hankel transform and construct an efficient and stable algorithm for its numerical evaluation and apply it to solve the realistic problem of inverse quasi-static steady-state thermal stresses in a thick circular plate, which is subjected to arbitrary interior temperature and determine the unknown temperature and thermal stresses.

\section{The Hankel Transform}

The general Hankel transform pair for Bessel function of order $v$ is defined as [1,2]

$$
H_{\nu}[f(r) ; p]=\int_{0}^{\infty} r f(r) J_{v}(p r) d r=F_{v}(p) .
$$

Hankel Transform is self reciprocal; its inverse is given by

$$
H_{v}^{-1}\left[F_{v}(p) ; r\right]=\int_{0}^{\infty} p F_{v}(p) J_{v}(p r) d p=f(r),
$$

where $J_{v}$ is the $v$ th-order Bessel function of first kind.

Several quality research articles have been published for the evaluation of Hankel transform. Analytical evaluations of (1) and (2) are rare and their numerical computations are difficult because of the oscillatory behaviour of the Bessel functions and infinite length of the interval involved in it. The efficiency of a method for computing HT is highly dependent on the function to be transformed and thus it is very difficult to choose an optimal algorithm for given function.

Postnikov [3], proposed for the first time, a novel and powerful method for computing zero and first order HT by using Haar wavelets. Refining the idea of Postnikov [3], Singh et al. [4-6] obtained three efficient algorithms for numerical evaluation of HT of order $v>-1$. All these algorithms depend on separating the integrand $r f(r) J_{v}(p r)$ into two components; the slowly varying components $r f(r)$ and the rapidly oscillating component $J_{v}(p r)$. Then either $r f(r)$ is expanded into various wavelet series using different orthonormal bases like Haar wavelets, linear Legendre multiwavelets, Fourier Bessel series and truncating the series at an optimal level or approximating $r f(r)$ by a quadratic over the subinterval using the Filon quadrature philosophy [7].

In this manuscript, we take an entirely different approach. Instead of manipulating the simpler component $r f(r)$, we manipulate the rapidly oscillating part $J_{v}(p r)$, thus avoiding the complexity of evaluating integrals involving Bessel functions. We use the hat basis functions described in "Hat Functions and Their Associated Properties" section, to approximate $J_{v}(p r)$ and replace it by its approximation in Eq. (1), thereby getting an efficient and stable algorithm for the numerical evaluation of the HT of order $v>-1$. In "Algorithm" section, we derive the algorithm and further give the error and the stability analysis in "Error and Stability Analysis" section. A numerical experiment to verify our theoretical findings is also provided in "Error and Stability Analysis" section. In "The Main Result" section, we apply our proposed algorithm to solve the realistic problem of inverse quasi-static steady-state thermal stresses in a thick circular plate, which is subjected to arbitrary interior temperature and determine the unknown temperature and thermal stresses on the upper surface of the thick 
circular plate, where the fixed circular edge and the lower surface of the circular plate are thermally insulated.

\section{Hat Functions and Their Associated Properties}

Hat functions are defined on the domain $[0,1]$. These are continuous functions with shape of hats, when plotted on two dimensional plane. The interval $[0,1]$ is divided into $n$ subintervals $[i h,(i+1) h], i=0,1,2, \ldots, n-1$, of equal lengths $h$ where $h=\frac{1}{n}$. The hat function's family of first $(n+1)$ hat functions is defined as follows [8]:

$$
\begin{aligned}
& \psi_{0}(t)= \begin{cases}\frac{h-t}{h}, & 0 \leq t<h, \\
0, & \text { otherwise },\end{cases} \\
& \psi_{i}(t)= \begin{cases}\frac{t-(i-1) h}{h}, & (i-1) h \leq t<i h, \\
\frac{(i+1) h-t}{h}, & \text { ih } \leq t<(i+1) h, i=1,2, \ldots, n-1, \\
0, & \text { otherwise, }\end{cases} \\
& \psi_{n}(t)= \begin{cases}\frac{t-(1-h)}{h}, & 1-h \leq t \leq 1, \\
0, & \text { otherwise. }\end{cases}
\end{aligned}
$$

From the definition of hat functions it is obvious that

$$
\psi_{i}(k h)= \begin{cases}1, & i=k \\ 0, & i \neq k\end{cases}
$$

The hat functions $\psi_{j}(t)$ are continuous, linearly independent and are in $L^{2}[0,1]$.

A function $f \in L^{2}[0,1]$ may be approximated as

$$
f(t) \simeq \sum_{i=0}^{i=n} f_{i} \psi_{i}(t)=f_{0} \psi_{0}(t)+f_{1} \psi_{1}(t)+f_{2} \psi_{2}(t)+\cdots+f_{n} \psi_{n}(t) .
$$

The important aspect of using extended hat functions in the approximation of function $f(t)$, lies in the fact that the coefficients $f_{i}$ in the Eq. (7), are given by

$$
f_{i}=f(i h), \quad \text { for } i=0,1,2 \ldots, n \text { where } h=1 / n .
$$

\section{Algorithm}

To derive the algorithm, we first assume that the domain space of input signal $f(r)$ extends over a limited region $0 \leq r \leq R$. From physical point of view, this assumption is reasonable due to the fact that the input signal $f(r)$ which represents the physical field is either zero or it has an infinitely long decaying tail out side a disc of finite radius- $R$. Therefore, in many practical applications either the input signal $f(r)$ has a compact support or for a given $\varepsilon>0$ there exists a positive real $R$ such that $\left|\int_{R}^{\infty} r f(r) J_{v}(p r) d r\right|<\varepsilon$, which is the case if $f(r)=o\left(r^{\lambda}\right)$, where $\lambda<-\frac{3}{2}$ as $r \rightarrow \infty$. Hence in either case, from Eq. (1), we have

$$
\hat{H}_{v}[f(r) ; p]=\int_{0}^{R} r f(r) J_{v}(p r) d r \equiv \hat{F}_{v}(p) .
$$


By scaling (9) may be written as

$$
\hat{F}_{v}(p)=\int_{0}^{1} r f(r) J_{v}(p r) d r
$$

which is known as finite Hankel transform (FHT). Equation (9) is a good approximation of the HT given by (2). Using Eqs. (7) and (8), $J_{v}(p r)$ may be approximated as

$$
J_{v}(p r) \simeq \sum_{i=0}^{n} J_{v}(p i h) \psi_{i}(r) .
$$

Using the approximation in (11), we get the algorithm to evaluate the Hankel transform as

$$
\begin{aligned}
\hat{F}_{v}(p) \simeq \int_{0}^{1} r f(r) \sum_{i=0}^{n} J_{v}(\text { pih }) \psi_{i}(r) d r \\
=\sum_{i=0}^{n} J_{v}(p i h) \int_{0}^{1} r f(r) \psi_{i}(r) d r \\
=J_{v}(0) \int_{0}^{h} r f(r) \psi_{0}(r) d r+\sum_{i=1}^{n-1} J_{v}(p i h) \int_{(i-1) h}^{(i+1) h} r f(r) \psi_{i}(r) d r \\
\\
+J_{v}(p) \int_{1-h}^{1} r f(r) \psi_{n}(r) d r .
\end{aligned}
$$

It is note worthy here that the integral $\int_{0}^{1} r f(r) \psi_{i}(r) d r$ appearing in Eq. (12), may be easily calculated as $f(r)$ is known function and $\psi_{i}(r)$ is a linear polynomial $\forall i$.

\section{Error and Stability Analysis}

Let the R.H.S. of (11) is denoted by $J_{v, n}(p r)$ i.e.

$$
J_{v, n}(p r)=\sum_{i=0}^{n} J_{v}(p i h) \psi_{i}(r)
$$

Now replacing $J_{v}(p r)$ in Eq. (10), we define an $n$th approximate $\hat{F}_{v, n}(p)$ of the FHT $\hat{F}_{v}(p)$ as follows:

Definition 4.1 An $n$th approximate finite Hankel transform of $f(r)$, denoted by $\hat{F}_{v, n}(p)$ is defined as

$$
\hat{F}_{\nu, n}(p)=\int_{0}^{1} r f(r) J_{v, n}(p r) d r=\sum_{i=0}^{n} J_{v}(p i h) \int_{0}^{1} r f(r) \psi_{i}(r) d r .
$$

Let $\varepsilon_{n}(p)$ denote the absolute error between the FHT $\hat{F}_{\nu}(p)$ and its $n$th approximate $\hat{F}_{v, n}(p)$, then we have the following: 
Theorem 4.1 If $J_{v}(p r)$ is approximated by the family of first $(n+1)$ hat functions as given in Eq. (11), then

(i) $\left|J_{v}(p j h)-J_{v, n}(p j h)\right|=0$, for $j=0,1,2, \ldots, n$.

(ii) $\left|J_{v}(p r)-J_{v, n}(p r)\right| \leq \frac{p^{2}}{2 n^{2}}+O\left(\frac{p^{3}}{n^{3}}\right)$, for $j h<r<(j+1) h, j=0,1,2, \ldots, n-1$.

(iii) $\varepsilon_{n}(p)=\left|\hat{F}_{v}(p)-\hat{F}_{v, n}(p)\right| \leq \frac{M p^{2}}{4 n^{2}}+O\left(\frac{p^{3}}{n^{3}}\right)$, where $|f(r)| \leq M$.

Proof See the Appendix 1.

The stability of the proposed algorithm is analyzed under the influence of noise. In what follows, the exact data function is denoted by $f(r)$ and the noisy data function $f^{\alpha}(r)$ is obtained by adding a random noise $\alpha$ to $f(r)$ such that $f^{\alpha}(r)=f(r)+\alpha \theta$, where $\theta$ is a uniform random variable with values in $[-1,1]$ such that $\left|f^{\alpha}(r)-f(r)\right| \leq \alpha$. Then we have:

Theorem 4.2 When the input signal $f(r)$ is corrupted with noise $\alpha$, the proposed algorithm reduces the noise at least by a factor of $1 / 2$ in the output data $\hat{F}_{v, n}$.

Proof See Apendix 2.

A test problem included in this section is solved with and without random perturbations (noises) to illustrate the efficiency and stability of proposed algorithm by choosing three different values of noise $\alpha$ as $\alpha_{0}=0, \alpha_{1}=0.002$ and $\alpha_{2}=0.005$.

The errors $E_{j}(p)$ (= the approximate FHT obtained from Eq. (12) with random noise $\alpha_{j}$-the exact FHT), $j=0,1,2$ are computed and their graphs are sketched, for different $n$. Further the parameter $p$ ranges between 0 to 30 in steps of 0.2. Figure 3 depicts the graph of $\left|\hat{F}_{v, n}^{\alpha}(p)-\hat{F}_{v, n}(p)\right|$ for the test function in example, which is in conformity with the Theorem 4.2. For this illustration, the computations are done in MATLAB 7.0.1 and the elapsed times in computations of FHT by CPU for MATLAB codes, are found to be $0.140,0.593,4.961$ and $69.545 \mathrm{~s}$ for $n=100,1000,10,000$ and 100,000 respectively. The least square errors $\left\|E_{j}(p)\right\|_{2}$ involved in computations of approximate FHT with noises $\alpha_{j}, j=0,1,2$ for the given example with $n=10,000$, are $1.0554 E-08,1.0554 E-08$ and $1.0554 E-08$. These are calculated, using the formula

$$
\left\|E_{j}(p)\right\|_{2}=\sqrt{\frac{\sum_{i=0}^{n} E_{j}^{2}\left(p_{i}\right)}{n+1},}
$$

where $p_{i}$ is taken in steps of 0.2 in the range $[0,30]$.

Example Consider the function $f(r)=\left(r^{2}-a^{2}\right)^{2}$ given in [9], whose zero order finite Hankel transform is given by $F_{0}(p)=\frac{8 a\left\{\left(8-a^{2} p^{2}\right) J_{1}(p a)-4 a p J_{0}(p a)\right\}}{p^{5}}$.

For numerical computation, we take $a=1$ to show comparison between exact HT $F_{0}(p)$ and $n$th approximate FHT $\hat{F}_{0, n}(p)$, in Fig. 1. The errors $E_{0}(p), E_{1}(p)$ and $E_{2}(p)$ for $n=100$ are shown in Fig. 2. Further Fig. 3 depicts the graph of $\left|\hat{F}_{0, n}^{\alpha}(p)-\hat{F}_{0, n}(p)\right|$, for noises $\alpha=0.002,0.005$ and random variable $\theta=0.2311$. Again it is to be noted here that in the caption of Fig. 3, $\left|\hat{F}_{0, n}^{\alpha}(p)-\hat{F}_{0, n}(p)\right|$ is denoted by $\left|H_{0}^{\alpha}(p)-H_{0}(p)\right|$. 


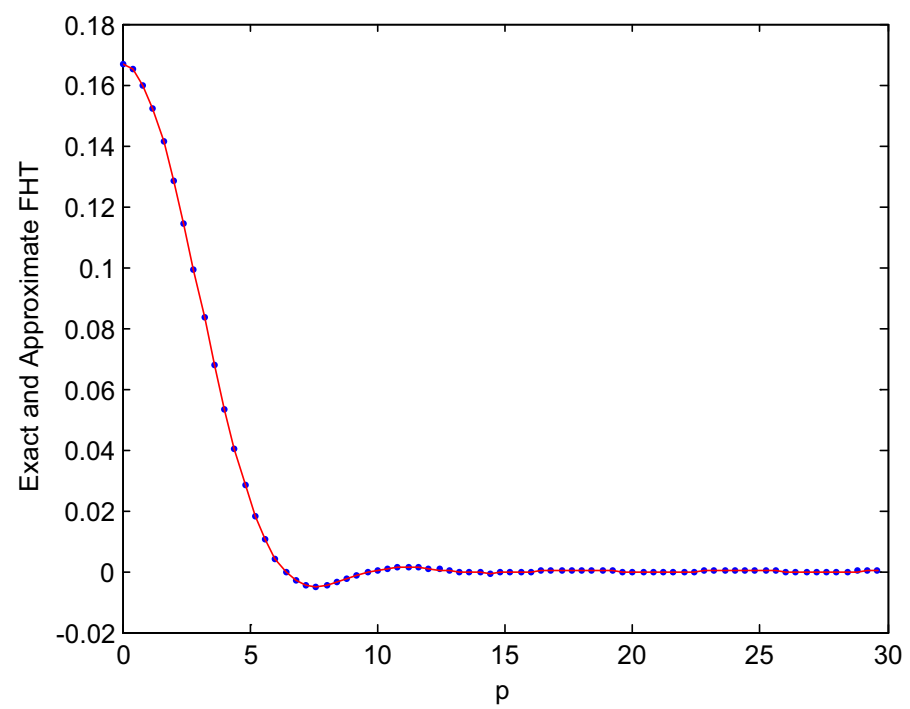

Fig. 1 The exact HT $F_{0}(p)$ (solid red line) and approximate HT $\hat{F}_{0, n}(p)$ (dotted line) for $n=100$

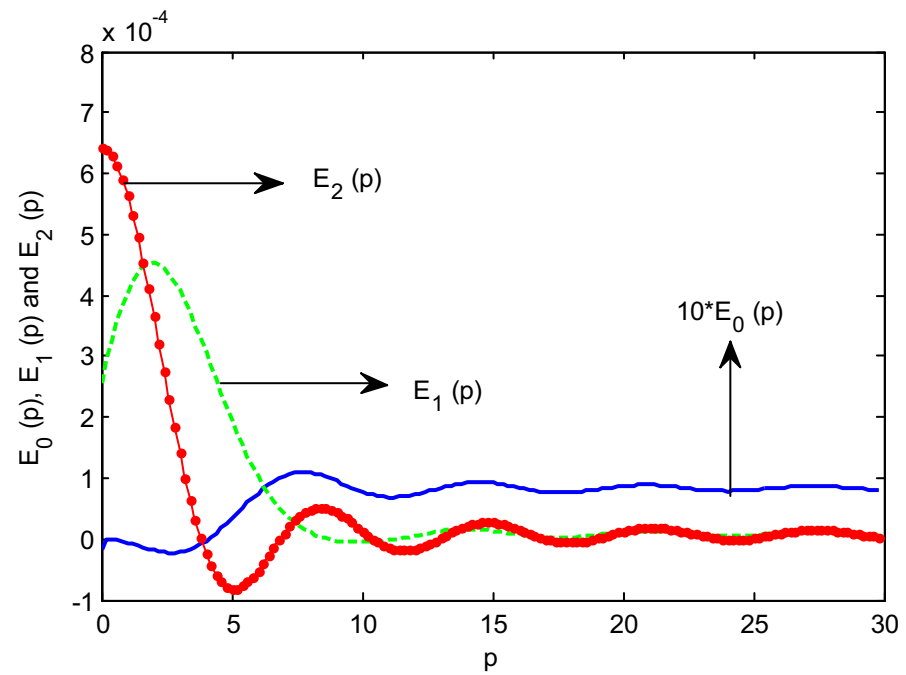

Fig. 2 Comparison of errors $E_{0}(p), E_{1}(p)$ and $E_{2}(p)$ for $n=100$

\section{The Main Result}

This section of the paper deals with the realistic problem of inverse quasi-static steady-state thermal stresses in a thick circular plate, which is subjected to arbitrary interior temperature. We apply the algorithm developed in "Algorithm" section, to determine the unknown temperature and thermal stresses on the upper surface of the thick circular plate, where the fixed circular edge and the lower surface of the circular plate are thermally insulated. The formulation of the problem is as follows [9]: 


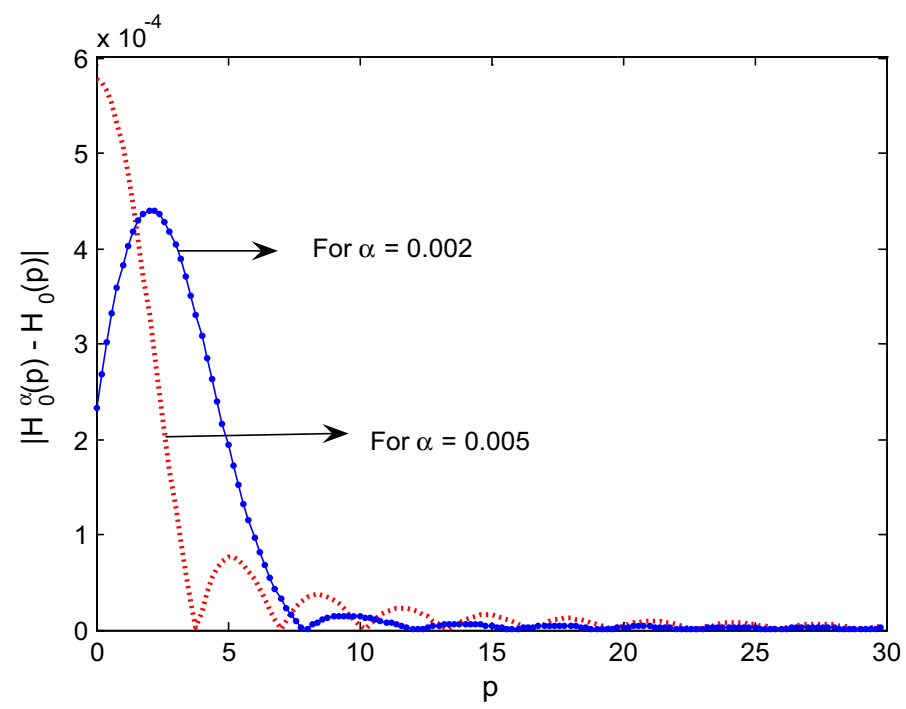

Fig. 3 Plot of $\left|H_{0}^{\alpha}(p)-H_{0}(p)\right|$ for $\alpha=0.002$ and $\alpha=0.005$ with $n=100$

We assume the thickness and radius of the circular plate to be $2 h$ and $a$ respectively. Let the plate be subjected to arbitrary known interior temperature $f(r)$ within the region $-h<z<h$.

Under these realistic conditions, the unknown temperature $g(r)$ and quasi-static thermal stresses due to unknown temperature $g(r)$ at the upper surface of plate $z=h$ are required to be determined.

The steady-state temperature of the disc satisfies the following heat conduction equation

$$
\frac{\partial^{2} T(r, z)}{\partial r^{2}}+\frac{1}{r} \frac{\partial T(r, z)}{\partial r}+\frac{\partial^{2} T(r, z)}{\partial z^{2}}=0,
$$

with the conditions

$$
\begin{aligned}
\frac{\partial T(r, z)}{\partial r} & =0 \text { at } r=a,-h \leq z \leq h, \\
\frac{\partial T(r, z)}{\partial z} & =0 \text { at } z=-h, 0 \leq r \leq a, \\
T(r, z) & =f(r) \text { (known) at } z=\xi,-h<\xi<h, 0 \leq r \leq a,
\end{aligned}
$$

and

$$
T(r, z)=g(r) \text { (unknown) at } z=h, 0 \leq r \leq a .
$$

The differential equation governing the displacement potential $\phi(r, z)$ is given in [10] as

$$
\frac{\partial^{2} \phi(r, z)}{\partial r^{2}}+\frac{1}{r} \frac{\partial \phi(r, z)}{\partial r}+\frac{\partial^{2} \phi(r, z)}{\partial z^{2}}=K \tau,
$$

where $K$ is the restraint coefficient and temperature change $\tau=T-T_{i}, T_{i}$ is the initial temperature. Displacement function is known as Goodier's thermoelastic displacement potential. 
The displacement functions in cylindrical coordinate system are represented by the Michell's function defined in [10] as

$$
\begin{aligned}
& u_{r}=\frac{\partial \phi}{\partial r}-\frac{\partial^{2} M}{\partial r \partial z}, \\
& u_{z}=\frac{\partial \phi}{\partial z}+2(1-s) \nabla^{2} M-\frac{\partial^{2} M}{\partial z^{2}} .
\end{aligned}
$$

The Michell's function $M$ must satisfy

$$
\nabla^{2} \nabla^{2} M=0
$$

where

$$
\nabla^{2}=\frac{\partial^{2}}{\partial r^{2}}+\frac{1}{r} \frac{\partial}{\partial r}+\frac{\partial^{2}}{\partial z^{2}}
$$

The component of the stresses are represented by the thermoelastic displacement potential $\phi$ and Michell's function $M$ as

$$
\begin{aligned}
& \sigma_{r r}=2 G\left[\frac{\partial^{2} \phi}{\partial r^{2}}-K \tau+\frac{\partial}{\partial z}\left(s \nabla^{2} M-\frac{\partial^{2} M}{\partial r^{2}}\right)\right], \\
& \sigma_{\theta \theta}=2 G\left[\frac{1}{r} \frac{\partial \phi}{\partial r}-K \tau+\frac{\partial}{\partial z}\left(s \nabla^{2} M-\frac{1}{r} \frac{\partial M}{\partial r}\right)\right], \\
& \sigma_{z z}=2 G\left[\frac{\partial^{2} \phi}{\partial r^{2}}-K \tau+\frac{\partial}{\partial z}\left(\left(2-s^{2}\right) \nabla^{2} M-\frac{\partial^{2} M}{\partial z^{2}}\right)\right]
\end{aligned}
$$

and

$$
\sigma_{r z}=2 G\left[\frac{\partial^{2} \phi}{\partial r \partial z}+\frac{\partial}{\partial r}\left((1-s) \nabla^{2} M-\frac{\partial^{2} M}{\partial z^{2}}\right)\right]
$$

where $G$ and $s$ are the shear modulus and Poisson's ratio, respectively.

For traction free surface the stress functions are

$$
\sigma_{r r}=\sigma_{r z}=0 \text { at } r=a \text { and } \sigma_{z z}=\sigma_{r z}=0 \text { at } z= \pm h \text {. }
$$

Equations (15)-(29) constitute mathematical formulation of the problem.

Using the finite Hankel transform and its inverse transform, Kulkarni and Deshmukh [9] gave the expression for temperature $T$ as follows:

$$
T(r, z)=\left(\frac{2}{a^{2}}\right) \sum_{n=1}^{\infty}\left[\frac{\bar{f}\left(\alpha_{n}\right) J_{0}\left(\alpha_{n} r\right)}{J_{0}^{2}\left(\alpha_{n} a\right)}\right]\left[\frac{\cosh \left[\alpha_{n}(z+h)\right]}{\cosh \left[\alpha_{n}(\xi+h)\right]}\right],
$$

where $\bar{f}\left(\alpha_{n}\right)$ is the Hankel transform of $f(r)$ and $\alpha_{1}, \alpha_{2}, \alpha_{3}, \ldots$ are roots of transcendental equation $J_{1}(\alpha a)=0$. Subsequently the expressions for unknown temperature $g(r)$ at upper surface $z=h$, displacements and stresses are given as [9]: 


$$
\begin{gathered}
g(r)=\left(\frac{2}{a^{2}}\right) \sum_{n=1}^{\infty}\left[\frac{\bar{f}\left(\alpha_{n}\right) J_{0}\left(\alpha_{n} r\right)}{J_{0}^{2}\left(\alpha_{n} a\right)}\right]\left[\frac{\cosh \left(2 \alpha_{n} h\right)}{\cosh \left[\alpha_{n}(\xi+h)\right]}\right] \\
u_{r}=\left(\frac{2 K}{a^{2}}\right) \sum_{n=1}^{\infty}\left[\frac{\bar{f}\left(\alpha_{n}\right) J_{1}\left(\alpha_{n} r\right)}{J_{0}^{2}\left(\alpha_{n} a\right)}\right]\left[\frac{(1-s) \cosh \left[\alpha_{n}(z+h)\right]}{\alpha_{n} \cosh \left[\alpha_{n}(\xi+h)\right]}\right], \\
u_{z}=\left(\frac{2 K}{a^{2}}\right) \sum_{n=1}^{\infty}\left[\frac{\bar{f}\left(\alpha_{n}\right) J_{0}\left(\alpha_{n} r\right)}{J_{0}^{2}\left(\alpha_{n} a\right)}\right]\left[\frac{(1-s) \sinh \left[\alpha_{n}(z+h)\right]}{\alpha_{n} \cosh \left[\alpha_{n}(\xi+h)\right]}\right] \\
\sigma_{r r}=\left(\frac{4 K G}{a^{2}}\right) \sum_{n=1}^{\infty}\left[\frac{\bar{f}\left(\alpha_{n}\right) J_{1}\left(\alpha_{n} r\right)}{r J_{0}^{2}\left(\alpha_{n} a\right)}\right]\left[\frac{(1-s) \cosh \left[\alpha_{n}(z+h)\right]}{\alpha_{n} \cosh \left[\alpha_{n}(\xi+h)\right]}\right] \\
\sigma_{\theta \theta}=\left(\frac{-4 K G}{a^{2}}\right) \sum_{n=1}^{\infty}\left[\frac{\bar{f}\left(\alpha_{n}\right)}{J_{0}^{2}\left(\alpha_{n} a\right)}\right]\left[\alpha_{n} J_{0}\left(\alpha_{n} r\right)-\frac{J_{1}\left(\alpha_{n} r\right)}{r}\right]\left[\frac{(1-s) \cosh \left[\alpha_{n}(z+h)\right]}{\alpha_{n} \cosh \left[\alpha_{n}(\xi+h)\right]}\right],
\end{gathered}
$$

$\sigma_{z z}=0$ and $\sigma_{r z}=0$.

As an application, let us consider the pair $f(r)=\left(r^{2}-a^{2}\right)^{2}$, for which the finite Hankel transform is given as [9]:

$$
\bar{f}\left(\alpha_{n}\right)=\frac{8 a\left[\left(8-a^{2} \alpha_{n}^{2}\right) J_{1}\left(\alpha_{n} a\right)-4 a \alpha_{n} J_{0}\left(\alpha_{n} a\right)\right]}{\alpha_{n}^{5}} .
$$

For numerical computation, the circular plate of steel (SN 50C) is considered. The parameters chosen for this application are $a=1 \mathrm{~m}, h=0.2 \mathrm{~m}$. The thermal diffusivity $k=15.9 \times$ $10^{6}\left(\mathrm{~m}^{2} \mathrm{~s}^{-1}\right)$ and Poisson ratio $s=0.281$. The series in the Eqs. (31-36) are truncated by taking first 10 positive roots (in increasing order) of transcendental equation $J_{1}(\alpha a)=0$, which are calculated by MATLAB subroutine "fzero" and are given as:

$$
\begin{aligned}
& \alpha_{1}=3.8317, \quad \alpha_{2}=7.0156, \quad \alpha_{3}=10.1735, \quad \alpha_{4}=13.3237, \quad \alpha_{5}=16.4706, \\
& \alpha_{6}=19.6159, \quad \alpha_{7}=22.7601, \quad \alpha_{8}=25.9037, \quad \alpha_{9}=29.0468, \quad \alpha_{10}=32.1897 .
\end{aligned}
$$

We set $A=\left(-16 / 10^{2} a\right), B=\left(16 K / 10^{2} a\right), C=\left(32 G K / 10^{2} a\right)$ for convenience to plot the graphs of $g(r) / A, u_{r} / B, u_{z} / B, \sigma_{r r} / C$ and $\sigma_{\theta \theta} / C$. The expressions for unknown temperature, displacement and stress components for this particular example are obtained by replacing $\bar{f}\left(\alpha_{n}\right)$ with $\hat{F}_{0}\left(\alpha_{n}\right)$ in Eqs. (31-35). In Eqs. (31-35), instead of exact $\bar{f}\left(\alpha_{n}\right)$ (as given in Eq. (36)), we use the approximate finite Hankel transform $\hat{F}_{0}\left(\alpha_{n}\right)$ obtained by the proposed algorithm in "Algorithm" section. For numerical computations of $u_{r}, u_{z}, \sigma_{r r}, \sigma_{\theta \theta}$ we take $z=h / 2$ and the plots of these quantities are drawn for different values of $\xi$ taken as $\xi=-0.2,-0.1,0,0.1$ and $0.2 \mathrm{~m}$. Further in the all the computations, first 1001 hat basis functions $\varphi_{0}, \varphi_{1}, \varphi_{2}, \ldots, \varphi_{1000}$ are used for finding approximate value of $\hat{F}_{0}$ at different $\alpha_{n}$. Numerical computations are done in MATLAB and variations of these quantities in radial directions are shown in the figures with help of MATLAB codes. The findings are discussed as follows:

In Fig. 4, the unknown temperature $g(r) / A$ is sketched for different $\xi$. This figure depicts that for $\xi=-0.2$ and $\xi=-0.1$, the tensile and compressive stresses are developed alternatively in the radial direction.

This typical behaviour of oscillatory nature of $g(r) / A$ in case of thick circular plate diminishes when we take a thin plate of height $h=0.02 \mathrm{~m}$, which is shown in Fig. 5. The sketch of $g(r) / A$ is shown in different multipliers to differentiate between different curves, otherwise the curves coincide. 


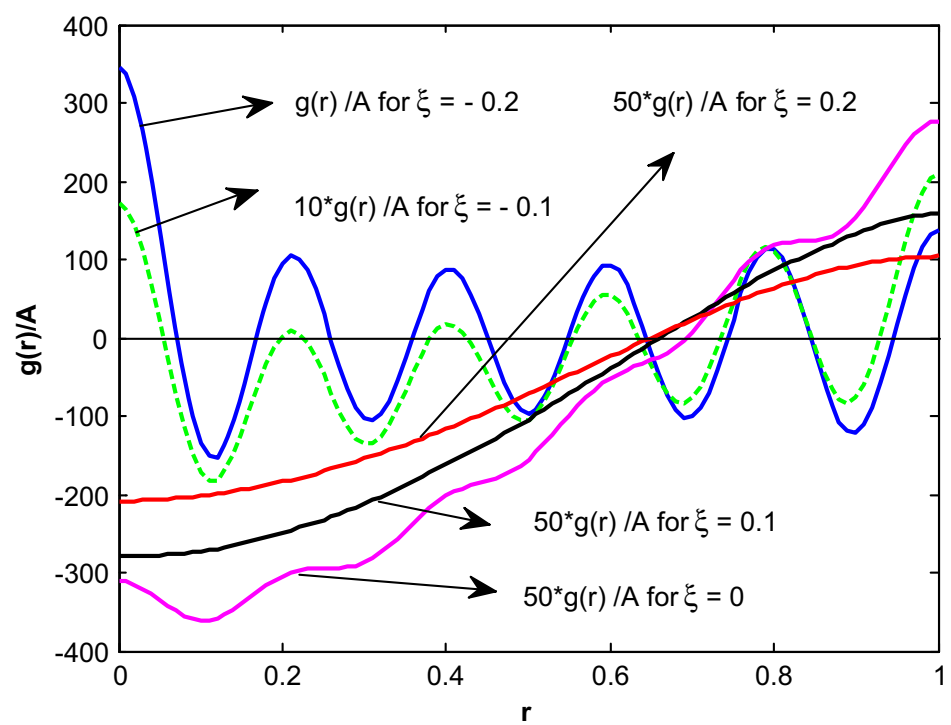

Fig. 4 The unknown temperature $g(r) / A$ in radial direction (thick plate, $h=0.2 \mathrm{~m}$ )

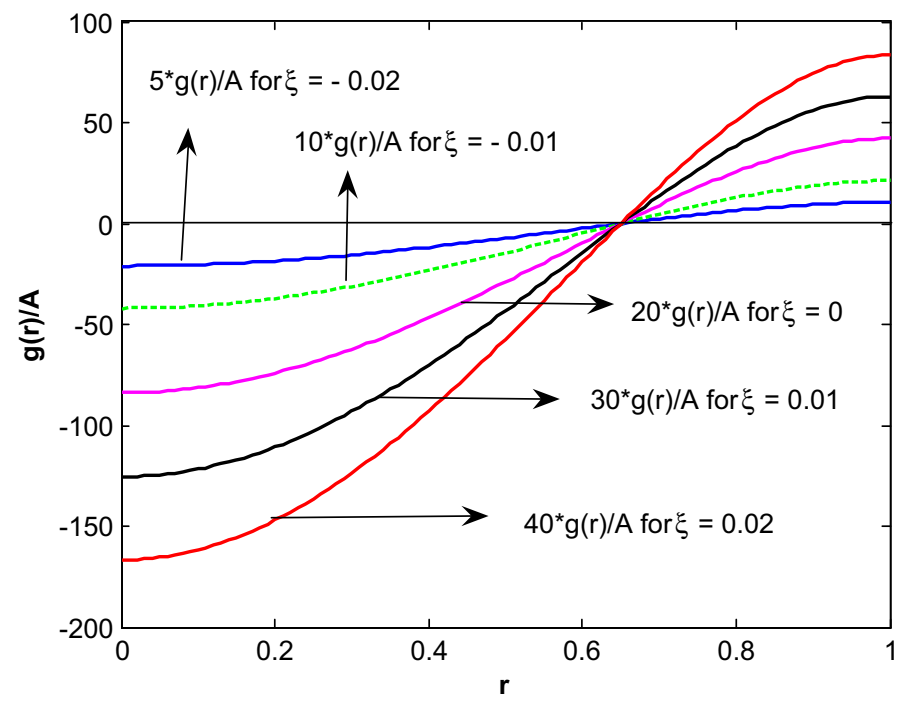

Fig. 5 The unknown temperature $g(r) / A$ in radial direction (thin plate $h=0.02 \mathrm{~m}$ )

The radial displacement $u_{r} / B$ is plotted in Fig. 6, which shows the normal curve and it is zero at $r=0$ and the circular boundary of the circular plate. Also it develops the tensile stress in the radial direction.

From Fig. 7, it is evident that the axial displacement $u_{z} / B$ develops tensile stress within circular region $0 \leq r \leq 0.7$ and compressive stress in annular region $0.7 \leq r \leq 1$.

From Fig. 8, it is observed that the radial stress $\sigma_{r r}$ shows compression in the region $0 \leq r \leq 0.1$, when heat source is near the lower surface $(\xi=-0.2)$. In other positions 


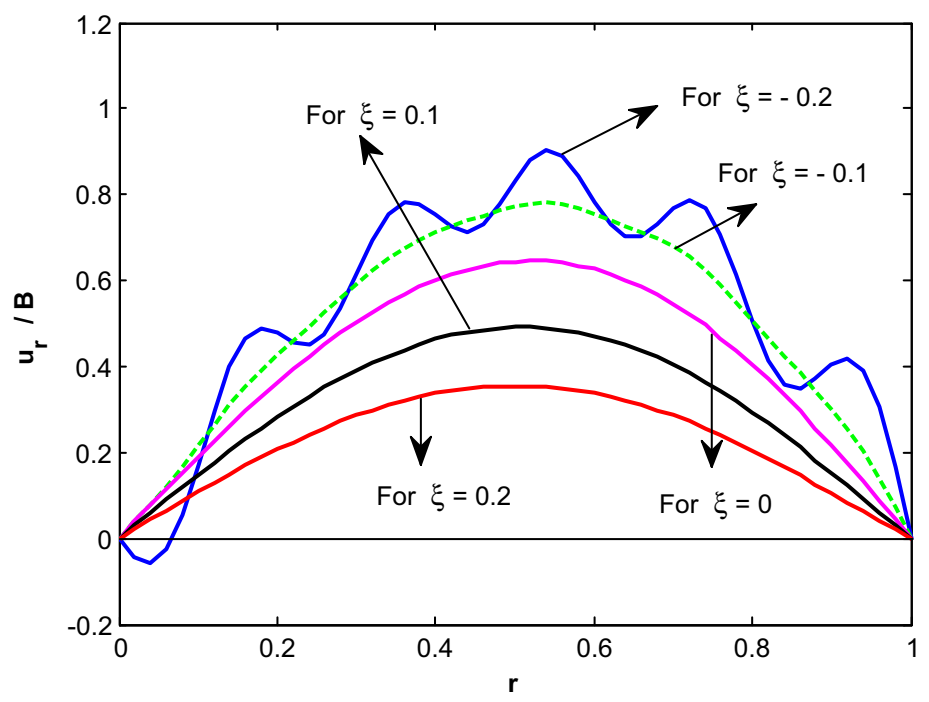

Fig. 6 The displacement function $u_{r} / B$ in radial direction

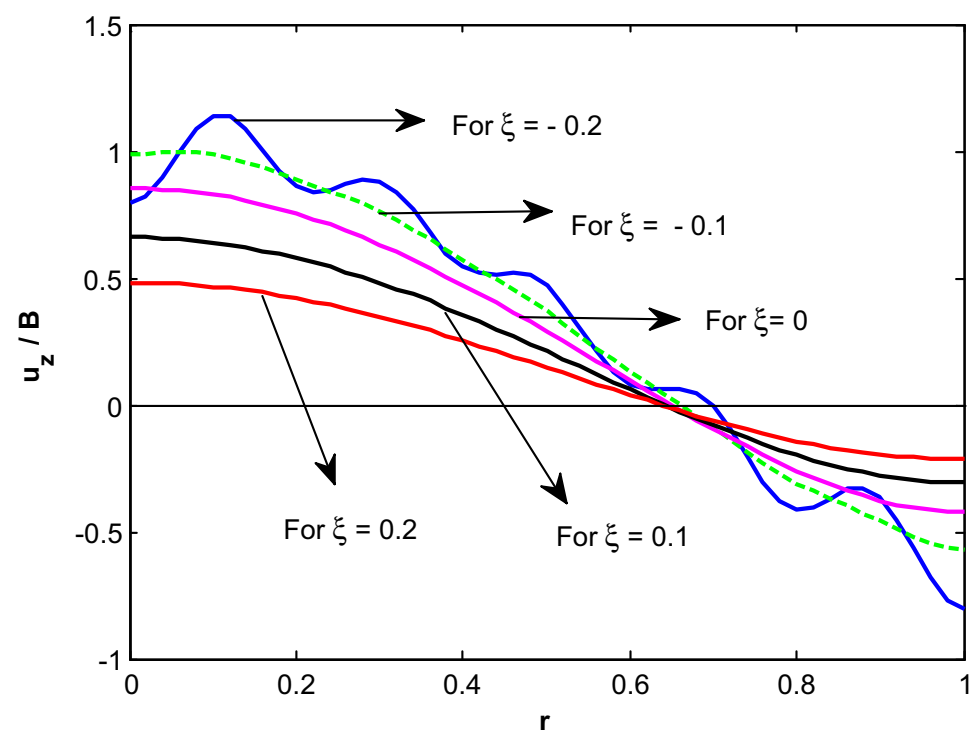

Fig. 7 The displacement function $u_{z} / B$ in radial direction

of heat source, the tensile nature of stress is seen. It is zero at the circular boundary of the circular plate.

From Fig. 9, the angular stress develops tensile and compressive stresses alternatively in case when heat source is near the lower surface. In other positions of heat source, the angular stress develops compressive stresses within circular region $0 \leq r \leq 0.5$ and tensile stress in annualar region $0.5<r \leq 1$ in the radial direction. 


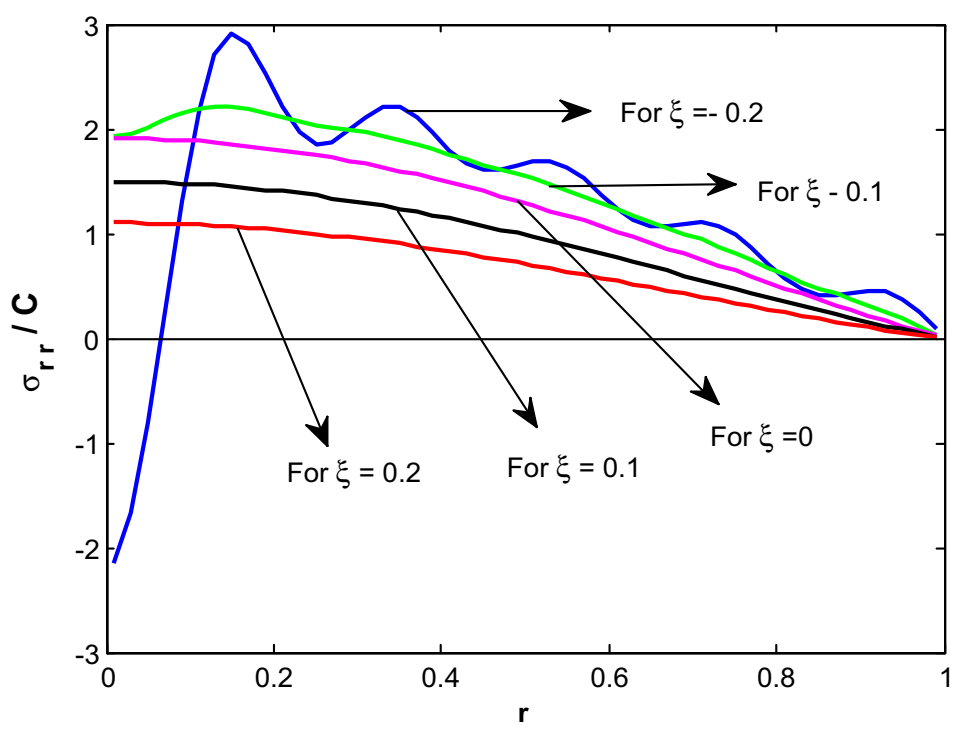

Fig. 8 The radial stress functions $\sigma_{r r} / C$ in radial direction

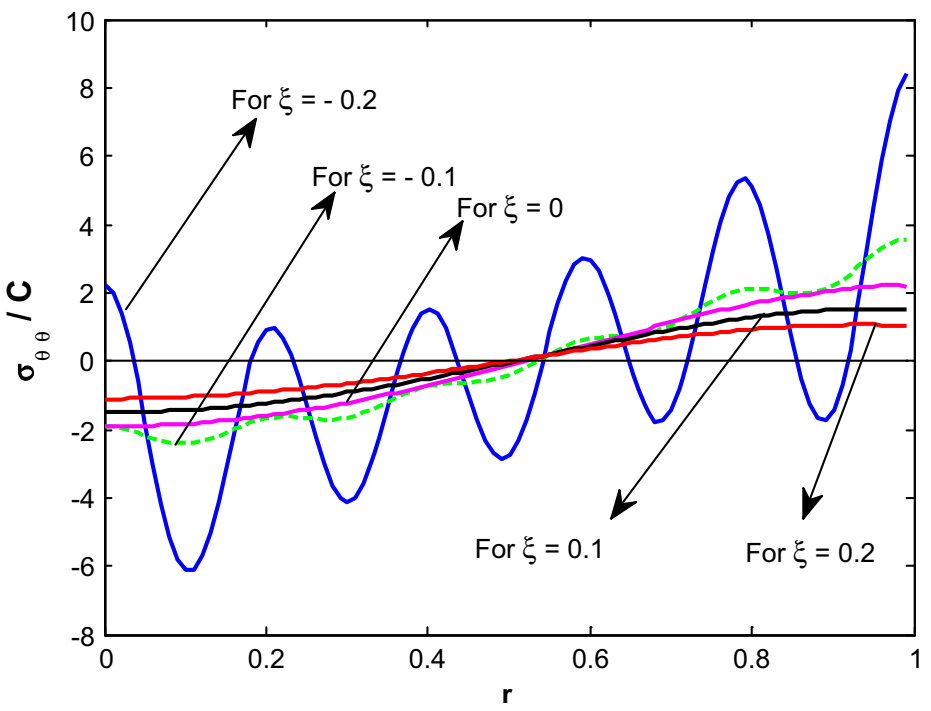

Fig. 9 The radial stress function $\sigma_{\theta \theta} / C$ in radial direction

Thus we are able to find the displacement and stress components which occur near heat source. With an increase in temperature the circular plate will tend to expand in the radial as well as in axial direction. In the plane state of stress the stress components $\sigma_{z z}$ and $\sigma_{r z}$ are zero. So it may be considered that due to unknown temperature the circular plate expands in the axial direction and bends concavely at the centre. This expansion is inversely proportional to the thickness of the circular plate. 


\section{Conclusions}

A new stable and efficient algorithm based on hat functions for the numerical evaluation of Hankel transform is proposed and analyzed. Replacing the rapidly oscillating part $J_{v}(p r)$ with its approximation by hat basis function avoids the complexity of evaluating integrals involving Bessel functions. This makes the evaluation of HT integral very simple. Choosing hat functions makes our algorithm attractive in their application in the applied physical problems as they eliminate the problems connected with the Gibbs phenomenon taking place in $[3,11]$. We have given error and stability analysis and by various numerical experiments have corroborated our theoretical findings. Stability with respect to the data is restored and excellent accuracy is obtained even for small sample interval and high noise levels in the data. From the various figures it is obvious that the algorithm is consistent and does not depend on the particular choice of the input signal. The accuracy and simplicity of the algorithm provides an edge over the others. Further, the algorithm is applied to solve the realistic problem of inverse quasi-static steady-state thermal stresses in a thick circular plate, which is subjected to arbitrary interior temperature and determine the unknown temperature and thermal stresses on the upper surface of the thick circular plate, where the fixed circular edge and the lower surface of the circular plate are thermally insulated. As a special case a mathematical model is numerically solved for $f(r)=\left(r^{2}-a^{2}\right)^{2}$. The thermoelastic behaviors such as temprature, displacements and stresses are examined with the help of arbitrary known interior temprature.

\section{Appendix 1}

Proof of Theoerm 4.1 (i) From Eqs. (13, "Error and Stability Analysis" section) and (6, "Hat Fucntions and Theri Associated Properties" section), the value of $J_{v}(p r)$ at $j$ th nodal point $r=j h, j=0,1,2, \ldots, n$ is given by

$$
\begin{aligned}
J_{v, n}(p j h) & =\sum_{i=0}^{n} J_{v}(p i h) \psi_{i}(j h)=J_{v}(p j h) \psi_{j}(j h)+J_{v}(p j h+p h) \psi_{j+1}(j h) \\
& =J_{v}(p j h) .
\end{aligned}
$$

So, $\left|J_{v}(p j h)-J_{v, n}(p j h)\right|=0$, for $j=0,1,2, \ldots, n$.

(ii) Further if $r$ lies between two consecutive integer multiples of $h$ i.e., $j h<r<(j+$ 1) $h, j=0,1,2, \ldots, n-1$, then from Eq. (13), we have

$$
\begin{aligned}
J_{v, n}(p r)= & J_{v}(p j h) \psi_{j}(r)+J_{v}(p j h+p h) \psi_{j+1}(r) \\
= & J_{v}(p j h)\left[\frac{(j+1) h-r}{h}\right] \\
& +J_{v}(p j h+p h)\left[\frac{r-j h}{h}\right](\text { using Eqs. (2.1-2.3) of section 2) } \\
= & J_{v}(p j h)-p j h\left[\frac{J_{v}(p j h+p h)-J_{v}(p j h)}{p h}\right] \\
& +p r\left[\frac{J_{v}(p j h+p h)-J_{v}(p j h)}{p h}\right]
\end{aligned}
$$

As $h \rightarrow 0$, from Eq. (37), we obtain

$$
J_{v, n}(p r) \simeq J_{v}(p j h)-p j h J_{v}^{\prime}(p j h)+p r J_{v}^{\prime}(p j h) .
$$


By expanding $J_{v}(p r)$ in form of Taylor's series in the powers of ( $\left.p r-p j h\right)$, we have

$$
J_{v}(p r)=\sum_{k=0}^{\infty} \frac{(p r-p j h)^{k}}{k !} J_{v}^{(k)}(p j h),
$$

where $J_{v}^{(k)}(p j h)$ is the $k$ th order derivative of $J_{v}(p r)$ w.r.t. $p r$ at $r=j h$. Using Eqs. (38) and (39), the error between exact and approximate values of $J_{v}(p r)$ is given by

$$
\begin{aligned}
J_{v}(p r)-J_{v, n}(p r) & =\sum_{k=2}^{\infty} \frac{(p r-p j h)^{k}}{k !} J_{v}^{(k)}(p j h) \\
& =\frac{(p r-p j h)^{2}}{2 !} J_{v}^{(2)}(p j h)+O(p r-p j h)^{3} .
\end{aligned}
$$

Since $(p r-p j h)<p h$ and $n h=1$, from Eq. (40), we get

$$
\left|J_{v}(p r)-J_{v, n}(p r)\right| \leq \frac{p^{2}}{2 n^{2}}\left|J_{v}^{\prime \prime}(p j h)\right|+O\left(\frac{p^{3}}{n^{3}}\right) .
$$

Since $J_{v}^{\prime}(x)=\frac{1}{2}\left[J_{v-1}(x)-J_{v+1}(x)\right]$, we have, $J_{v}^{\prime \prime}(p j h)=\frac{1}{4}\left[J_{v-2}(p j h)-2 J_{v}(p j h)\right.$ $\left.+J_{v+1}(p j h)\right]$, and hence

$$
\begin{aligned}
\left|J_{v}(p r)-J_{v, n}(p r)\right| & \leq \frac{p^{2}}{8 n^{2}}\left|J_{v-2}(p j h)-2 J_{v}(p j h)+J_{v+1}(p j h)\right|+O\left(\frac{p^{3}}{n^{3}}\right) \\
& \leq \frac{p^{2}}{2 n^{2}}+O\left(\frac{p^{3}}{n^{3}}\right), \text { as }\left|J_{v}(p j h)\right| \leq 1
\end{aligned}
$$

(iii) The absolute error $\varepsilon_{n}(p)$ between exact FHT $\hat{F}_{v}(p)$ and its $n$th approximate $\hat{F}_{v, n}(p)$, is given by

$$
\begin{aligned}
\varepsilon_{n}(p)= & \left|\hat{F}_{v}(p)-\hat{F}_{v, n}(p)\right|=\left|\int_{0}^{1} r f(r)\left(J_{v}(p r)-J_{v, n}(p r)\right) d r\right| \\
& =\left|\sum_{j=0}^{n-1} \int_{j h}^{(j+1) h} r f(r)\left(J_{v}(p r)-J_{v, n}(p r)\right) d r\right| \\
& \leq \sum_{j=0}^{n-1} \int_{j h}^{(j+1) h} r|f(r)|\left|\left(J_{v}(p r)-J_{v, n}(p r)\right)\right| d r \\
& \leq\left[\frac{p^{2}}{2 n^{2}}+O\left(\frac{p^{3}}{n^{3}}\right)\right] \sum_{j=0}^{n-1} \int_{j h}^{(j+1) h} r|f(r)| d r, \text { (follows from Eq. (6)). }
\end{aligned}
$$

If $|f(r)| \leq M$, we have

$$
\varepsilon_{n}(p) \leq M\left[\frac{p^{2}}{2 n^{2}}+O\left(\frac{p^{3}}{n^{3}}\right)\right] \sum_{j=0}^{n-1} \int_{j h}^{(j+1) h} r d r=\frac{M p^{2}}{4 n^{2}}+O\left(\frac{p^{3}}{n^{3}}\right) .
$$




\section{Appendix 2}

Proof of Theorem 4.2 If the approximate finite Hankel transform of the perturbed function $f^{\alpha}(r)$ is denoted by $\hat{F}_{v, n}^{\alpha}(p)$ then from Eq. (14, "Error and Stability Analysis" section)

$$
\hat{F}_{\nu, n}^{\alpha}(p)=\int_{0}^{1} r f^{\alpha}(r) \sum_{i=0}^{n} J_{v}(p i h) \psi_{i}(r) d r=\sum_{i=0}^{n} J_{v}(p i h) \int_{0}^{1} r f^{\alpha}(r) \psi_{i}(r) d r .
$$

From Eqs. (14) and (43), we have

$$
\begin{aligned}
\left|\hat{F}_{\nu, n}^{\alpha}(p)-\hat{F}_{v, n}(p)\right| & =\mid \sum_{i=0}^{n} J_{v}(\text { pih }) \int_{0}^{1} r f^{\alpha}(r) \psi_{i}(r) d r-\sum_{i=0}^{n} J_{v}(\text { pih }) \int_{0}^{1} r f(r) \psi_{i}(r) d r \mid \\
& =\mid \sum_{i=0}^{n} J_{v}(\text { pih }) \int_{0}^{1} r\left(f^{\alpha}(r)-f(r)\right) \psi_{i}(r) d r \mid \\
& \leq \sum_{i=0}^{n} \mid J_{v}(\text { pih })\left|\int_{0}^{1} r\right|\left(f^{\alpha}(r)-f(r)\right)|| \psi_{i}(r) \mid d r \\
& \leq \sum_{i=0}^{n}\left|J_{v}(p i h)\right| \int_{0}^{1} r|\alpha \theta|\left|\psi_{i}(r)\right| d r \\
& \leq \sum_{i=0}^{n} \int_{0}^{1} r|\alpha \theta| \psi_{i}(r) d r, \text { as }\left|J_{v}(p i h)\right| \leq 1 \& \psi_{i}(r) \geq 0 .
\end{aligned}
$$

Hence, substituting $\psi_{i}(r)$ from Eqs. (3-5, "Hat Functions and Theri Associated Properties" section), we get

$$
\begin{aligned}
\left|\hat{F}_{\nu, n}^{\alpha}(p)-\hat{F}_{\nu, n}(p)\right| \leq & |\alpha \theta|\left[\int_{0}^{h} r \frac{(h-r)}{h} d r+\sum_{i=1}^{n-1}\left\{\int_{(i-1) h}^{i h} r \frac{(r-(i-1) h)}{h} d r\right.\right. \\
& \left.\left.+\int_{i h}^{(i+1) h} r \frac{((i+1) h-r)}{h} d r\right\}+\int_{1-h}^{1} r \frac{(r-(1-h))}{h} d r\right] \\
= & |\alpha \theta|\left[\frac{h^{2}}{6}+h^{2} \sum_{i=1}^{n-1} i+\frac{\left(3 h-h^{2}\right)}{6}\right] \leq \frac{|\alpha|}{2},(\text { using }|\theta| \leq 1 \text { and } n h=1) .
\end{aligned}
$$

\section{References}

1. Sneddon, I.N.: The Use of Integral Transforms. Mc Graw-Hill, New York (1972)

2. Bracewell, R.: The Fourier Transform and Its Application. Mc Graw-Hill, New York (1965)

3. Postnikov, E.B.: About calculation of the Hankel transform using preliminary wavelet transform. J. Appl. Math. 6, 319-325 (2003) 
4. Singh, V.K., Singh, O.P., Pandey, R.K.: Numerical evaluation of Hankel transform by using linear Legendre multi-wavelets. Comput. Phys. Commun. 179, 424-429 (2008)

5. Singh, V.K., Singh, O.P., Pandey, R.K.: Efficient algorithms to compute Hankel transforms using wavelets. Comput. Phys. Commun. 179, 812-818 (2008)

6. Singh, V.K., Pandey, R.K., Saurabh, Singh: A stable algorithm for Hankel transforms using hybrid of Block-pulse and Legendre polynomials. Comput. Phys. Commun. 181, 1-10 (2010)

7. Barakat, R., Parshall, E., Sandler, B.H.: Zero-order Hankel transform algorithms based on Filon quadrature philosophy for diffraction optics and beam propogation. J. Opt. Soc. Am. A 15, 652-659 (1998)

8. Babolian, E., Mordad, M.: A numerical method for solving systems of linear and nonlinear integral equations of the second kind by hat basis functions. Comput. Math. Appl. 62(1), 187-198 (2011)

9. Kulkarni, V.S., Deshmukh, K.C.: An inverse quasi-static steady state in a thick circular plate. J. Frank1. Inst. 345(1), 29-38 (2008)

10. Noda, N., Hetnarsky, R.B., Tanigawa, Y.: Thermal Stresses, 2nd edn. Taylor \& Francis, New York (2003)

11. Guizar-Sicairos, M., Gutierrez-Vega, J.C.: Computation of quasi-discrete Hankel transforms of integer order for propagating optical wave fields. J. Opt. Soc. Am. A 21(1), 53 (2004) 